\title{
Déterminants Sociotechniques De L'élevage De La Volaille Au Sein Des Concessions Dans La Commune De Bambey, Sénégal
}

\author{
Abdou Khadre Fall, \\ Université Alioune Diop (UAD), Institut Supérieur de Formation Agricole et Rurale \\ (ISFAR, ex ENCR), Département Productions Animales Bambey Sénégal \\ Thierry Daniel Tamsir Nesseim , \\ Université Iba Der Thiam de Thiès (UIDT), Ecole Nationale Supérieure \\ d’Agriculture (ENSA), Thiès, Sénégal

\section{Sokhna Diarra Ndour,} \\ Université Alioune Diop (UAD), Institut Supérieur de Formation Agricole et Rurale \\ (ISFAR, ex ENCR), Département Productions Animales Bambey Sénégal
}

Doi:10.19044/esj.2021.v17n40p158

Submitted: 03 May 2021

Accepted: 28 September 2021

Published: 30 November 2021
Copyright 2021 Author(s)

Under Creative Commons BY-NC-ND 4.0 OPEN ACCESS

Cite As:

Fall A.K., Nesseim T.D.T., \& Ndour S.D (2021). Déterminants Sociotechniques De L'élevage De La Volaille Au Sein Des Concessions Dans La Commune De Bambey, Sénégal. European Scientific Journal, ESJ, 17(40), 158.

https://doi.org/10.19044/esj.2021.v17n40p158

\section{Résumé}

L'aviculture est une activité socio-économique très répandue et importante au Sénégal car c'est un secteur qui pourvoie des emplois. L'étude s'est déroulée auprès de 150 aviculteurs à travers une enquête formelle dans la commune de Bambey, région de Diourbel au Sénégal. Les enquêtes ont porté sur les caractéristiques socio-économiques des éleveurs, la conduite technique et économique des élevages mais aussi sur l'analyse des contraintes. Elle est constituée de $70,7 \%$ d'hommes et $29,3 \%$ de femmes dont $75 \%$ sont mariés, $19 \%$ sont célibataires et $6 \%$ sont veufs avec une moyenne d'âge de $38,6 \pm 13,5$ ans. L'activité est exercée notamment par des fonctionnaires (22,7\%), des étudiants (17 ,3\%), des commerçants, des ménagères et des ouvriers à $14,7 \%$ pour chaque groupe. Ceux dont l'aviculture est leur activité principale ne représentent que $8 \%$. La motivation des aviculteurs est plus qu'économique $(53,3 \%)$ et passionnelle $(25,3 \%)$. L'appropriation de la volaille revient à $88 \%$ des enquêtés, à 6,7\% aux membres de la famille et à 4\% 
aux enfants. Les élevages sont de type mono spécifique (52\%) et plurispécifique (48\%). Les espèces élevées sont les poulets (locaux et exotiques), les pigeons, les canards, les dindes, les oies, les pintades et les cailles. La quasi-totalité des éleveurs $(73,3 \%)$ alimentent leurs volailles au moins une fois par jour alors que 26,7\% laissent leurs animaux en divagation. La reproduction est mieux contrôlée chez les espèces de races exotiques. La vaccination est pratiquée par $64,6 \%$ des enquêtés. Les pathologies dominantes sont la maladie de Newcastle $(47,6 \%)$, le parasitisme $(18,4 \%)$, la variole $(13,4 \%)$ et le coryza (10,3\%), Les principales contraintes sont les maladies, la cherté des aliments, la chaleur, le manque de formation. Cependant, le développement de l'aviculture nécessite la mise en place d'un programme de conseil. La subvention et l'implication de tous les acteurs du système est nécessaire pour son développement.

Mots clés: Aviculture, Reproduction, Caractéristiques, Typologie, Pathologie

\title{
Socio-Technical Determinants of Poultry Rearing Within Concessions in the Commune of Bambey, Senegal
}

\author{
Abdou Khadre Fall, \\ Université Alioune Diop (UAD), Institut Supérieur de Formation Agricole et Rurale \\ (ISFAR, ex ENCR), Département Productions Animales Bambey Sénégal \\ Thierry Daniel Tamsir Nesseim, \\ Université Iba Der Thiam de Thiès (UIDT), Ecole Nationale Supérieure \\ d’Agriculture (ENSA), Thiès, Sénégal \\ Sokhna Diarra Ndour, \\ Université Alioune Diop (UAD), Institut Supérieur de Formation Agricole et Rurale \\ (ISFAR, ex ENCR), Département Productions Animales Bambey Sénégal
}

\begin{abstract}
Poultry farming is a very widespread and important socio-economic activity in Senegal because it is a sector that provides jobs. The study involved 150 poultry farmers through a formal survey in the commune of Bambey, Diourbel region in Senegal. The surveys focused on the socio-economic characteristics of the farmers, the technical and economic management of the farms, and the analysis of constraints. It is made up of $70.7 \%$ men and $29.3 \%$ women, $75 \%$ of whom are married, $19 \%$ are single and $6 \%$ are widowed, with an average age of $38.6 \pm 13.5$ years. The activity is carried out by civil servants (22.7\%), students (17.3\%), shopkeepers, householders and workers (14.7\% for each group). Those whose main activity is poultry farming represent only $8 \%$. The motivation of poultry farmers is more than economic (53.3\%) and passionate (25.3\%). The ownership of the animals belongs to $88 \%$ of the
\end{abstract}


respondents, $6.7 \%$ to family members and $4 \%$ to children. The farms are mono-specific (52\%) and multi-specific (48\%). The species raised are chickens (local and exotic), pigeons, ducks, turkeys, geese, guinea fowl and quails. Almost all farmers (73.3\%) feed their poultry at least once a day while $26.7 \%$ let their animals roam. Reproduction is better controlled in exotic breeds. Vaccination is practiced by $64.6 \%$ of respondents. The dominant diseases are Newcastle disease (47.6\%), parasitism (18.4\%), smallpox (13.4\%) and coryza (10.3\%). The main constraints are diseases, the high cost of food, heat and lack of training. However, the development of poultry farming requires the establishment of an advisory program. The subsidy and involvement of all the actors in the system is necessary for its development.

Keywords: Poultry Farming, Reproduction, Characteristics, Typology, Pathology

\section{Introduction}

La filière avicole est en plein essor au Sénégal depuis 2005 avec l'arrêt de l'importation de produits avicoles, suite à l'avènement de l'Influenza Aviaire Hautement Pathogène (IAHP). La filière avicole est en plein essor au Sénégal depuis 2005 avec l'arrêt de l'importation de produits avicoles, suite à l'avènement de l'Influenza Aviaire Hautement Pathogène (IAHP). La production intensive de volaille s'est considérablement développée ces dernières décennies, notamment, dans les zones urbaines et péri urbaines. L'élevage des espèces à cycle court, plus particulièrement, l'aviculture, est notée de plus en plus dans les milieux urbains comme un moyen pour améliorer la situation socio-économique des populations (Fall, 2016 ; Fall, 2017 ; Fall et Mbengue, 2021). En effet, l'élevage des espèces à cycle court est certainement une des solutions pour améliorer la couverture en protéines des populations. Il s'agit également d'élevages qui se rentabilisent très vite. Parmi ces espèces, la volaille constitue celle dont l'élevage demande peu de moyens et il peut être pratiqué par toutes les catégories socio-professionnelles. Cette volaille constitue une des rares opportunités d'épargne, d'investissement et de protection contre le risque (Sonaiya et Swan, 2004).Le secteur avicole y a contribué à hauteur de 19\% au PIB de l'Elevage en 2018 (PNDE, 2017). Son chiffre d'affaires global a été de près de 150 milliards de FCFA avec une production de 50 millions de poulets et un investissement de plus de 20 milliards de FCFA. Il génère plus de 50000 emplois directs et indirects (MEPA, 2019).

L'effectif de la volaille en 2018 était de 81418550 de sujets dont la volaille industrielle représente $65,18 \%$ des effectifs. Il y a eu une hausse de $11,0 \%$ de la volaille industrielle entre 2016 et 2018. Quant à la volaille traditionnelle, elle n’a augmenté que de 3,5\% (ANSD, 2019). 
La production d'œufs de consommation en 2017 a été estimée à 719 millions d'unités, soit un taux de réalisation de $4 \%$ de plus par rapport à l'objectif de 694 millions d'unités qui était visé. Elle a cependant évolué de 6\% par rapport à l'année 2016, correspondant à un volume de 44 millions d'unités. ${ }^{1}$

La filière avicole est en plein essor au Sénégal depuis 2005 avec l'arrêt de l'importation de produits avicoles, suite à l'avènement de l'Influenza Aviaire Hautement Pathogène (IAHP). La production intensive de volaille s'est considérablement développée ces dernières décennies, notamment, dans les zones urbaines et péri urbaines.

L'élevage des espèces à cycle court, plus particulièrement, l'aviculture, est notée de plus en plus dans les milieux urbains comme un moyen pour améliorer la situation socio-économique des populations (Fall, 2016 ; Fall, 2017 ; Fall et Mbengue, 2021). En effet, l'élevage des espèces à cycle court est certainement une des solutions pour améliorer la couverture en protéines des populations. Il s'agit également d'élevages qui se rentabilisent très vite. Parmi ces espèces, la volaille constitue celle dont l'élevage demande peu de moyens et il peut être pratiqué par toutes les catégories socioprofessionnelles. Cette volaille constitue une des rares opportunités d'épargne, d'investissement et de protection contre le risque (Sonaiya et Swan, 2004) de vulnérabilité en milieu rural.

L'enquête a pour but de contribuer à la production d'informations sociotechniques sur la conduite de l'aviculture urbaine au niveau du Sénégal et plus particulièrement dans la commune de Bambey, région de Diourbel.

\section{Matériel et méthode}

\subsection{Zone d'étude}

Située à $14^{\circ} 42$ de latitude nord et $16^{\circ} 27$ de longitude ouest, la commune de Bambey est le chef-lieu du département éponyme. Cette commune de la région administrative de Diourbel a une superficie de $4,1 \mathrm{~km}^{2}$ et une population projetée à 34787 habitants en 2019 (ANSD, 2016) dont 53,03\% de femmes. Elle est caractérisée par sa jeunesse ; en effet, $44,8 \%$ de la population ont moins de 15 ans. Les moins de 20 ans font environ 55,7\% et seulement 3,5\% ont 65 ans et plus.

La population est dominée par les ethnies Wolofs (60\%) et Sérères (25\%). Les 15\% autres sont les Diolas, les Manjacks, les Sarakholés, les Bambaras, les Maures, les Halpulars. Les langues parlées sont principalement: le Wolof $(72 \%)$, le Sérère (15,7\%), le Halpular (10,2\%) et autres (2,1\%). Les activités économiques dominantes à Bambey sont constituées principalement par le commerce et l'agriculture.

\footnotetext{
${ }^{1}$ Ministère de l’Elevage et des Productions Animales - Sénégal Rapport de revue sectorielle 2018 version finale
} 


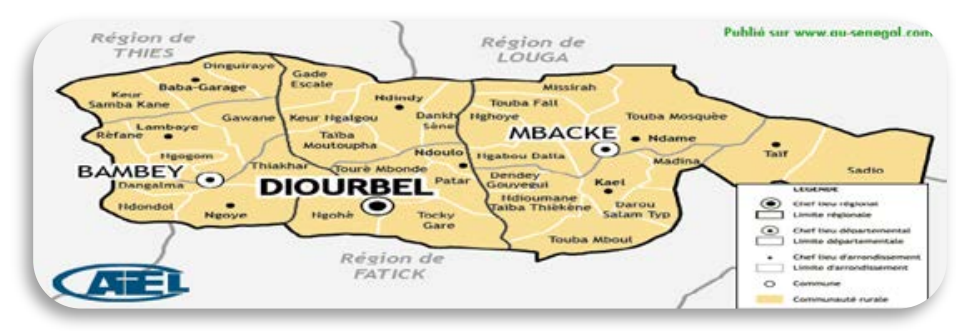

Figure 1: limites administratives et position du département de Bambey

(Source AVSF, 2016)

\subsection{Collecte et traitement des données}

La collecte des données a commencé par l'identification et le recensement des éleveurs au niveau des différents quartiers de la commune. Ce recensement a permis d'identifier les éleveurs de volaille dans leur concession qui sont au nombre de 197. Tous les éleveurs accessibles, volontaires et disponibles, au nombre de 150, ont été soumis à un questionnaire par le biais d'une enquête formelle.

Les caractéristiques socio-économiques des éleveurs recherchées étaient : le sexe, l'âge, l'ethnie, le niveau d'études, la situation matrimoniale, la profession, la raison d'élevage, la religion, la formation reçue, la durée dans l'activité et l'origine des animaux. Les données zootechniques recherchées étaient : les oiseaux élevés, la race, le type de production, l'effectif actuel, le taux de mortalité, les pathologies rencontrées, la gestion de la reproduction, l'alimentation et la charge de travail. Les données économiques de l'élevage à identifier étaient : les différentes charges, les recettes

Les données collectées ont été traitées avec le logiciel Excel 2013 et SPHINX (matrice de dépouillement) avant d'être analysées par le logiciel SPSS, version IBM SPSS Statistic 20 (analyse descriptive, tableau de croisé dynamique, moyenne, écart type, fréquence, minima, maxima, test de $\mathrm{X}^{2}$ d'indépendance sur tableaux croisés).

Une analyse SWOT a été réalisée pour synthétiser les résultats en termes de forces, de faiblesses, d'opportunités et de menaces qui existent au niveau de l'élevage de la volaille.

\section{Résultats et discussion}

\subsection{Résultats}

Les résultats issus de l'analyse et du traitement des données collectées se présentent comme suit: caractéristiques socio-économiques des éleveurs, la conduite technique et économique des élevages et l'identification des différentes contraintes. 


\subsubsection{Caractéristiques socio-économiques des éleveurs}

Les caractéristiques socio-économiques ont concerné le genre, la situation matrimoniale, l'âge, la religion, l'ethnie, le niveau d'instruction, les activités principales et secondaires, la durée dans l'activité, la formation reçue et les raisons d'élevage.

Il ressort de l'analyse que l'aviculture est majoritairement pratiquée par des hommes (70,7\%) plus que les femmes (29,30\%). Les éleveurs enquêtés sont constitués de mariés (75\%), de célibataires (19\%) et de veufs $(6 \%)$. Le genre a une influence sur la situation matrimoniale $\left(X^{2}=0,021\right)$.

L'âge moyen est de $38,6 \pm 13,5$ ans. Il est compris entre 15 et 75 ans. Il est à noter que $85,3 \%$ des aviculteurs ont entre 15 et 50 ans. Cependant seul $4 \%$ ont un âge égal ou supérieur à 70 ans. Les hommes ont un âge compris entre 15 et 75 ans avec une moyenne de 38,6 $\pm 13,5$ ans tandis que celui des femmes est de $39 \pm 13,7$ ans avec un minimum de 20 ans et un maximum de 68 ans.

\subsubsection{Religion, ethnie et niveau d'instruction}

Les éleveurs enquêtés sont caractérisés par la diversité de leur religion, de leur ethnie et et de leur niveau d'instruction.Ils sont des musulmans (94,7\%) et des chrétiens (5,3\%). Les ethnies rencontrées sont Wolofs (41,3\%), Sérères (29,3\%) e Halpulaars (20\%), Mandjack (4\%), Maure (4\%) et Diola (1,3\%).

Les éleveurs ont différents niveaux d'instruction: universitaires (20\%), élémentaires (20\%), secondaires (17,3\%) et moyens (12\%). Cependant, 17,3\% sont des analphabètes et les $13,3 \%$ ont fait des études coraniques.

Les hommes ont fait des études universitaires (26,4\%), secondaires (22,6\%), élémentaires (20,8\%), coraniques (15,1\%), moyennes (9,4\%). Les hommes analphabètes ne sont que $5,6 \%$ tandis que les femmes sont à $45,5 \%$. Cependant, certaines ont fréquenté le cycle moyen (22,7\%), primaire (13,6\%), coranique $(9,1)$, secondaire $(4,6 \%)$ et universitaire $(4,6 \%)$. Le genre est fortement corrélé au niveau d'instruction $\left(\mathrm{X}^{2}=0,00\right)$.

\subsubsection{Activités principales et secondaires}

La situation professionnelle des éleveurs est illustrée par la figure figure 4.

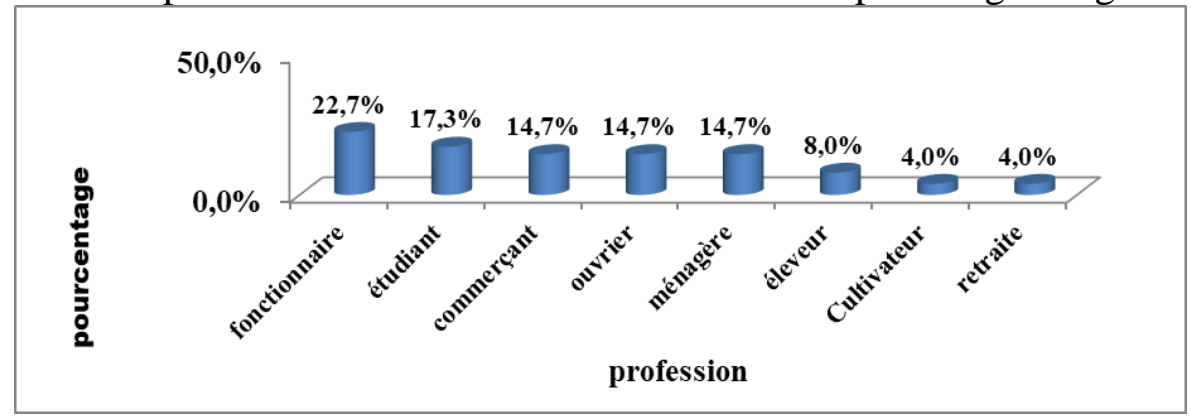

Figure 1 : répartition des aviculteurs selon leur profession (sources : nos enquêtes) 
L'analyse de cette figure révèle que $22,7 \%$ sont des fonctionnaires suivis par les étudiants (17,3\%). Il n'y a que 8,0\% des éleveurs qui ont une activité principale. Le genre a une forte corrélation avec l'activité principale $\left(\mathrm{X}^{2}=0,00\right)$.

De façon globale, $81,3 \%$ des enquêtés s'adonnent à l'aviculture comme activité secondaire.

\subsubsection{Ancienneté, formation reçue, raisons d'élevage}

L'ancienneté des éleveurs dans l'activité avicole varie entre 1 et 50 ans avec une moyenne de 12,9 $\pm 12,8$ ans. Seulement, $8 \%$ des enquêtés ont une fois reçu une formation en aviculture.

La durée dans l'activité d'élevage des femmes est de 10 12,1 ans tandis que chez les hommes, elle est de 13,2 $\pm 13,2$ ans. L'aviculture est essentiellement exercée pour des raisons économiques (53,3\%) et de passion $(25,3 \%)$ mais certains éleveurs le font pour la consommation familiale (12\%), par tradition $(9,3 \%)$. Le genre n'influe pas sur les motivations $\left(X^{2}=0,132\right)$ des éleveurs.

La volaille appartient à l'enquêté qui peut être le chef ou un membre de la famille (88\%), à la famille entière ou propriété collective (6,7\%) et aux enfants $(5,3 \%)$. Le genre a une influence sur l'appropriation des animaux $\left(X^{2}=0,039\right)$.

\subsection{Caractéristiques techniques}

\subsubsection{Typologie des élevages}

La typologie montre un élevage mono spécifique ou présence d'une seule espèce de volaille (52\%) et d'un élevage plurispécifique qui est la combinaison de plusieurs espèces de volaille (48\%).

$\mathrm{Au}$ niveau des élevages mono spécifiques, ceux qui font uniquement des poulets de chairs représentent $69,2 \%$ (tableau 1 ).

Les élevages plurispécifiques ont 2, 3, 4, 5 et 6 espèces de volailles. Ceux qui ont deux espèces de volaille représentent $52,8 \%$ de leur groupe (tableau 1 ).

Tableau $1:$ typologie des élevages

\begin{tabular}{ccccc}
\hline Typologie & composition & nombres & \% dans le groupe & \% échantillons \\
\hline Mono & poulets de chairs & 54 & $69,20 \%$ & 36,0 \\
spécifique & poulets locaux ou exotiques & 16 & $20,50 \%$ & 10,7 \\
& Pigeons & 8 & $10,30 \%$ & 5,3 \\
pluri & sous total & $\mathbf{7 8}$ & $\mathbf{1 0 0 , 0 0 \%}$ & $\mathbf{5 2 , 0}$ \\
spécifique & à deux espèces & 38 & $52,80 \%$ & 25,3 \\
& à trois espèces & 12 & $16,70 \%$ & 8,0 \\
& à quatre espèces & 8 & $11,10 \%$ & 5,3 \\
& à cinq espèces & 6 & $8,30 \%$ & 4,0 \\
& à six espèces & 8 & $11,10 \%$ & 5,3 \\
& sous total & $\mathbf{7 2}$ & $\mathbf{1 0 0 , 0 0 \%}$ & $\mathbf{4 8 , 0}$ \\
\hline
\end{tabular}


(source: nos enquêtes)

\subsubsection{Espèces élevées}

Plusieurs espèces de volaille ont été identifiées (tableau 2). Il s’agit des poulets, des pigeons, des canards, des dindes, des oies, des pintades et des cailles.

Le poulet (local et/ou exotique) est présent chez 68 aviculteurs soit $90,7 \%$ des enquêtés. Les élevages de poulet de chair représentent $36 \%$ de l'échantillon et $69,2 \%$ des exploitations mono spécifiques. Les élevages de poulets font $54,7 \%$ des enquêtés avec une représentation de $20,5 \%$ des élevages mono spécifiques et $91,7 \%$ des élevages plurispécifiques.

Les pigeons (colombiculture) sont rencontrés dans $40 \%$ de l'échantillon. Ils sont présents dans 10,3\% des élevages mono spécifiques et $72,2 \%$ des élevages plurispécifiques.

Les élevages de canards sont seulement rencontrés dans les exploitations plurispécifiques (50\%) alors que dans l'échantillon, ils ne sont présents que dans $24 \%$ des aviculteurs.

L'élevage de l'oie n'est présent que dans les élevages plurispécifiques (36,11\%). Les éleveurs qui en élèvent représentent 17,3\% de l'échantillon total.

Les pintades sont présentes dans les élevages à plusieurs espèces soit $30 \%$ de ce groupe et $14,7 \%$ de l'échantillon total.

L'élevage de dindes n'est rencontré que dans 19,4\% des plurispécifiques et occupe 9,3\% des enquêtés. Les cailles ne sont présentes que dans une seule exploitation soit $2 \%$ et $1,3 \%$ de l'échantillon.

Tableau 2 : espèces élevées

\begin{tabular}{|c|c|c|c|c|c|}
\hline Typologie & Espèces élevées & nombre & total & $\begin{array}{l}\text { \% dans le } \\
\text { groupe }\end{array}$ & $\begin{array}{c}\% \text { dans } \\
\text { l'échantillon }\end{array}$ \\
\hline Mono & poulets de chair & 54 & & $69,2 \%$ & $36,0 \%$ \\
\hline \multirow{2}{*}{ spécifique } & poulets locaux ou exotiques & 16 & & $20,5 \%$ & $10,7 \%$ \\
\hline & Pigeons & 8 & & $10,3 \%$ & $5,3 \%$ \\
\hline sous total & & & 72 & $100,0 \%$ & $52,0 \%$ \\
\hline \multirow[t]{8}{*}{ à 2 espèces } & poulets/pigeons & 22 & 38 & $52,8 \%$ & $25,3 \%$ \\
\hline & poulets/canards & 4 & & & \\
\hline & poulets/dindes & 2 & & & \\
\hline & poulets/oies & 2 & & & \\
\hline & poulets/pintades & 2 & & & \\
\hline & canards/oies & 2 & & & \\
\hline & pigeons/oies & 2 & & & \\
\hline & pigeons/canards & 2 & & & \\
\hline \multirow[t]{4}{*}{ à 3 espèces } & poulets /canards/pigeon & 4 & 12 & $16,7 \%$ & $8,0 \%$ \\
\hline & poulets/pintades/pigeons & 4 & & & \\
\hline & poulets/oies/pigeons & 2 & & & \\
\hline & poulets/canards/oies & 2 & & & \\
\hline
\end{tabular}




\begin{tabular}{|c|c|c|c|c|c|}
\hline à 4 espèces & $\begin{array}{l}\text { poulets/canard/pigeon/dinde } \\
\text { poulet/canard/dinde/pintade } \\
\text { poulet/canard/pigeon/oie } \\
\text { poulet/canard/pintade/oie }\end{array}$ & $\begin{array}{l}2 \\
2 \\
2 \\
2\end{array}$ & 8 & $11,1 \%$ & $5,3 \%$ \\
\hline à 5 espèces & $\begin{array}{c}\text { poulet/canard/pintade/oie/ } \\
\text { pigeon } \\
\text { poulet/canard/dinde/pintade/ } \\
\text { pigeon }\end{array}$ & 4 & 6 & $8,3 \%$ & $4,0 \%$ \\
\hline à 6 espèces & $\begin{array}{c}\text { poulet/canard/dinde/pintade/oie/pigeo } \\
\mathrm{n}\end{array}$ & 6 & 8 & $11,1 \%$ & $5,3 \%$ \\
\hline $\begin{array}{c}\text { sous total } \\
\text { Totaux }\end{array}$ & pigeon/caille & & $\begin{array}{c}36 \\
150 \\
\end{array}$ & $100,0 \%$ & $\begin{array}{c}48,0 \% \\
100,0 \% \\
\end{array}$ \\
\hline
\end{tabular}

\subsubsection{Races et effectifs}

(Source: nos enquêtes)

Les poulets sont élevés par 90,6\% des exploitations enquêtées et sont de différentes races. Les poulets de chair sont présents dans 18,7\% des élevages. Les lots varient de 50 à 2900 sujets par bande avec une moyenne de 232,8 sujets. La plupart des producteurs de poulets de chair (59,3\%) font une production continue durant toute l'année alors que 33,3\% parmi eux ciblent les fêtes religieuses. Cependant, 7,4\% des éleveurs arrêtent leurs productions durant l'hivernage.

Les poulets locaux existent dans les exploitations de $17,6 \%$ des enquêtés. L'effectif moyen des sujets est de 10,2 $\pm 7,5$ et il est compris entre 1 et 40 sujets.

Les races exotiques de poule sont rencontrées dans 10,1 \% des élevages. Le nombre de poules « dites de race » par exploitant varie de 4 à 40 avec une moyenne de 11,6 $6 \pm 8,9$. Les races exotiques et/ou ornementales de poulets identifiés sont au nombre de 8 (figure 3 ).

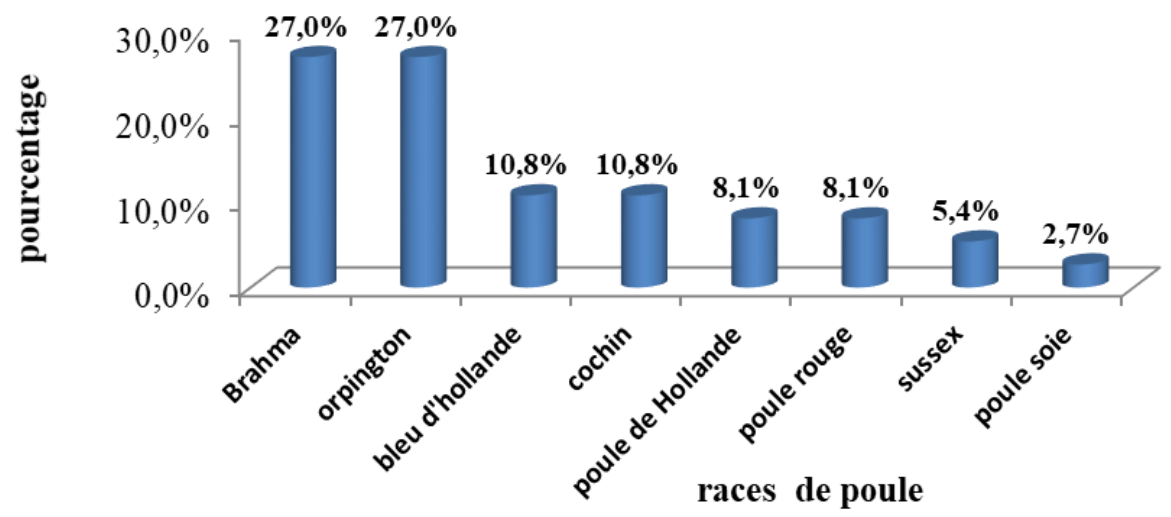

Figure 2: Répartition des races de poules (source: nos enquêtes) 
Il ressort de cette étude que les races Brahmas et Orpingtons sont les plus présentes soit $27 \%$ chacune, suivis des bleus de Hollande et Cochin (10,8\% chacun). Les poules de Hollande et poules rouges ont aussi le même pourcentage de représentativité (soit 8,1\%). Les races qui sont les moins présentées sont le Sussex $(5,4 \%)$ et la poule soie $(2,7 \%)$.Plusieurs races de pigeon sont présentes dans la commune de Bambey. Les pigeons voyageurs (22,5\%), les locaux (18,6\%) et les Boulants (7,8\%) sont les plus fréquents. Les races King, Lahore, Beauté allemand et Mondain ont le même pourcentage (3,9\%). Les 39,4\% qui restent sont principalement constitués de Cauchois, Château Batam, Paon, Texan, Gouvret, Capucin, Colombe, Cravaté, Manteau, métis (bâtard), Poule florentine, Romain, Tambour, Dragon, Gazzi, Polonais et Messager de Beyrouth. Les effectifs des pigeons vont de 2 à 100 paires

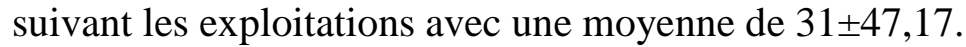

Trois races de canards ont été signalées par les éleveurs. La race locale ou ordinaire représente $72,2 \%$ des effectifs des élevages. Les deux autres races sont le canard de chair (22,2\%) et le colvert (soit 5,6\%). Les effectifs se situent entre 2 et 40 canards suivant les élevages avec une moyenne de 12,7 $\pm 11,6$.

Trois races d'oies sont exploitées. Il s'agit de l'oie de Guinée (46,2\%), de l'oie française (38,5\%) et de l'oie de Toulouse (15,4\%). Leur effectif varie de 2 à 8 avec une moyenne de $3,5 \pm 1,8$.

Les éleveurs de pintades exploitent principalement la race locale (58,3\%) et les pintades de chair $(41,7 \%)$. Les effectifs des pintades sont compris entre 1 à 30 avec une moyenne de 10,8 $\pm 8,8$.

Les dindes et les cailles sont dites "de races locales". L'effectif des dindes

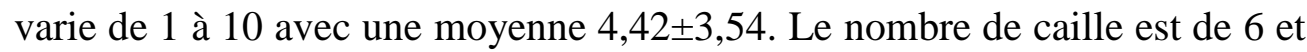
un seul éleveur le pratique.

\subsubsection{Mode d'acquisition, habitat et équipements}

Toutes les espèces de volaille élevées ont été achetées au début des activités d'élevage. Les poulets de chair sont achetés au niveau des magasins (36\%) de revendeurs. Les autres sujets proviennent soit du marché réservé à la vente de volaille ou "Daraal Guinaar"(49,3\%) soit du voisinage (14,7\%). La totalité des éleveurs enquêtés possède des logements ou poulaillers pour leurs volailles. Les poulaillers sont faits en dur (36\%) ou avec du matériel de récupération comme les tôles en zinc, du grillage et/ou du bois (64\%). Les poulaillers sont localisés au niveau de la cour, de la terrasse et des coins des concessions.

En dehors des élevages de poulets de chair, certains éleveurs possèdent une poussinière $(31,3 \%)$.

Les équipements sont constitués de mangeoires (artisanales (28\%, bols et plats usés), d'abreuvoirs (modernes, bidons recoupés etc.), de couveuses, de râteaux, de balais, de pelles, de sceaux et de congélateurs. 


\subsubsection{Alimentation et main d'œuvre}

Différents types d'aliments sont utilisés par les éleveurs pour nourrir la volaille . Certains éleveurs (38,7\%) utilisent des aliments de type industriels, tandis que d'autres (28\%) utilisent un mélange fait à base de céréales locales comme le mil, le sorgho, le maïs, le blé, le son de mil ou le niébé pour les poulets et pigeons, l'arachide et petit pois pour les pigeons, le son de riz pour les canards.

La quasi-totalité des éleveurs (73,3\%) alimentent leurs volailles au moins une fois par jour alors que 26,7\% laissent leurs animaux en divagation. La distribution de l'aliment aux volailles se fait le matin et le soir $(70,7 \%$ des enquêtés), le matin (20\%), le matin, à midi et le soir (9,3\%) selon éleveurs interviewés. C'est l'eau de robinet qui est utilisée pour abreuver la volaille. La main-d'œuvre utilisée provient de la famille.

\subsubsection{Pratiques de reproduction et de sélection}

Les pratiques de reproduction varient suivant la conduite des espèces et des races. Au niveau des poulets exotiques, 60,5\% des éleveurs ne contrôlent pas la reproduction, 36,8\% font des croisements dont 2,6\% concernent la race locale et Brahma ou local et Orpington.

Les éleveurs de pigeons (45,2\%) veillent aux accouplements afin d'éviter des croisements.

La reproduction des canards, pintades, oies, dindes et cailles n'est pas contrôlée. La couvaison de leurs œufs se fait de façon naturelle ou à l'aide d'une couveuse (37\%).

La couvaison des œufs de la volaille locale (poulets, pigeons, canards, dindes) se fait par leur propre femelle.

Au niveau des poulets dits de race, la couvaison des œufs est faite par les poules locales (44,4\%), par leurs propres femelles (29\%), au niveau des couveuses $(18,9 \%)$ et par les canes $(7,7 \%)$.

Les éleveurs de pigeons dits de race ont recours aux pigeons ordinaires ou locaux (11,5\%), aux voyageurs $(11,5 \%)$ et aux métisses ou bâtards (3,8\%) pour la couvaison des œufs.

Au niveau des pintades, ils ont recours aux poules locales $(22,2 \%)$ et aux couveuses $(11,1 \%)$.

Quant à la couvaison des œufs d'oies, 45,5\% des éleveurs utilisent des couveuses, tandis que $36,4 \%$ ont plutôt recours aux canes et $9,1 \%$ aux poules locales.

\subsubsection{Hygiène et de santé}

\subsubsection{Chez les poulets de chair}

Tous les éleveurs de poulets de chair utilisent de la litière. Le copeau de bois est utilisé par 66,7\% des éleveurs alors que les 33,3\% préfèrent la balle de riz. 
Le nettoyage systématique des matériels d'élevage est fait tous les $7 \pm 2$ jours. Le vide sanitaire est une pratique chez tous les aviculteurs. Cependant, $12 \%$ des aviculteurs le font pour une durée de 15 jours tandis que chez 9,3\%, le temps est compris entre 3 et 10 jours. Il est à noter que l'intervalle entre deux bandes peut varier, chez $78,7 \%$ des éleveurs, entre 21 et 90 jours.

Le calendrier vaccinal n'est pas systématique. Seuls 87,8\% des éleveurs vaccinent leurs sujets (maladie de Gumboro, maladie de Newcastle) alors les $22,3 \%$ utilisent des vitamines et des antibiotiques pour protéger leurs volailles.

Les pathologies rencontrées sont surtout la coccidiose $(44,4 \%)$ et la maladie de Gumboro (29,6\%). Cependant, 22,2\% de ces éleveurs disent n’avoir jamais constaté de maladies.

\subsubsection{Chez les autres volailles}

Les principales affections rencontrées sont la maladie de Newcastle $(47,6 \%)$, le parasitisme $(18,4 \%)$, la variole $(13,4 \%)$, le coryza $(10,3 \%)$, la salmonellose (6,2\%), la coccidiose $(4,1 \%)$ Cependant, $18,8 \%$ des éleveurs n’ont pas constaté de maladies au sein de leur élevage.

La fréquence de nettoyage des locaux par mois est en moyenne 2,5 $\pm 3,6$. La vaccination est pratiquée que par 35,4\% des éleveurs. 50\% des éleveurs qui font la vaccination ignorent la pathologie concernée. La vaccination contre la maladie de Newcastle est pratiquée par $8,3 \%$ des éleveurs. Seuls 6,3\% vaccinent contre la variole.

Les éleveurs (82,4\%) ont recours aux antibiotiques, vitamines, anticoccidiens et vermifuges. Cependant, $11,8 \%$ utilisent des produits vétérinaires dont ils ignorent les noms. Certains (5,9\%) utilisent des traitements traditionnels comme le piment, le tabac, l'écorce du fromager, l'ail, les feuilles de Moringa olifera, le café, les feuilles d'Accacia nilotica (Nép Nép), l’Aloe vera pour traiter des pathologies.

\subsection{Contraintes rencontrées}

\subsubsection{Chez les poulets de chair}

Les contraintes majeures auxquelles la production de poulets de chair est confrontée est la cherté de l'aliment industriel associé à des poussins de race ou espèce mauvaise selon les éleveurs. Les pathologies comme la maladie de Gumboro, la coccidiose et la salmonellose sont récurrentes dans cette zone. Les contraintes majeures auxquelles la production de poulets de chair est la cherté de l'aliment associé à des poussins de qualité inférieure selon les éleveurs. Les pathologies comme la maladie de Gumboro, la coccidiose et la salmonellose sont récurrentes dans cette zone.

La chaleur (température) qui sévit dans le milieu constitue également un véritable fléau pour le développement de l'aviculture 


\subsubsection{Chez les autres volailles}

$\mathrm{Au}$ niveau des poulets locaux, exotiques et/ou d'ornement, des pigeons, des pintades, des oies, des canards, des dindes et des cailles, les principales contraintes déclarées sont liées à la présence de maladies (variole, coryza, la maladie de Newcastle, salmonellose et coccidiose), les charges alimentaires, les prédateurs (vautours) et le vol.

Le manque de formation des aviculteurs est aussi un problème qui a été signalé. Il en est de même pour la présence de spécialiste en aviculture dans la zone, comme l'affirment les éleveurs. La divagation de certains animaux à longueur de journée est une réalité entrainant parfois des prédations.

L'absence de foire pour l'exposition des différentes races élevées et le manque de clients sont aussi des problèmes qui gangrènent l'élevage de la volaille. L'analyse des forces, faiblesses, opportunités et menaces (tableau 3) permet de mieux appréhender les différentes facettes de l'élevage de la volaille.

\section{Tableau 3: analyse SWOT}

\begin{tabular}{|c|c|}
\hline Forces & Faiblesses \\
\hline $\begin{array}{l}\text { - } \begin{array}{c}\text { diversité des espèces et races } \\
\text { élevées }\end{array} \\
\text { certains élevages ne demandent } \\
\text { pas un gros investissement } \\
\text { cycle d'élevage court } \\
\text { sources de revenus } \\
\text { supplémentaires pour les } \\
\text { pluriactifs } \\
\text { rôles social, culturel et sécuritaire } \\
\text { indéniable } \\
\text { utilisation des objets de } \\
\text { récupération pour l'équipement } \\
\text { valorisation des déchets } \\
\text { alimentaires domestiques } \\
\text { sources de protéines pour la } \\
\text { famille } \\
\text { Opportunités } \\
\text { marché hebdomadaire du lundi et } \\
\text { du vendredi } \\
\text { Existence de marchés et foires à } \\
\text { Dakar, Thiès et Touba pour la } \\
\text { volaille exotique } \\
\text { Présence du Département des } \\
\text { productions animales de } \\
\text { l’Université de Bambey, du } \\
\text { Centre Régional des Euvres } \\
\text { Universitaires et Sociales et du } \\
\text { Centre National de Recherche } \\
\text { Agronomique comme marché } \\
\text { potentiel }\end{array}$ & $\begin{array}{cc}\text { - } & \text { prix des aliments élevés } \\
\text { - } & \text { non diversité des aliments } \\
\text { - } & \text { manque de formation des } \\
\text { éleveurs } \\
\text { - } \\
\text { - } \quad \text { divence et persingatance de maladies de la volaires } \\
\text { absence d'organisation des } \\
\text { aviculteurs }\end{array}$ \\
\hline
\end{tabular}




\subsection{Discussion}

La discussion des résultats concerne les caractéristiques socioéconomiques des aviculteurs, les caractéristiques économiques et les contraintes.

\subsubsection{Caractéristiques socio-économiques des aviculteurs}

L'aviculture est une activité qui est pratiquée par des hommes et des femmes d'âge, d'ethnie et de profession différents.

Les éleveurs de volaille sont surtout des hommes contrairement à l'aviculture familiale qui est plus pratiquée, en milieu rural, surtout par les femmes et les enfants (Diallo., 2018). La forte présence des hommes est notée au niveau de l'élevage des poulets de chair et des pigeons qui est plus lucratif. Cela peut s'expliquer par le coût assez élevé de ces deux types d'élevage (prix des poussins, pigeons, aliment) et du fait qu'en ville les femmes ont peu de moyens financiers car ce sont le plus souvent des ménagères. Ces résultats sont confirmés au niveau de la commune de Thiès (Fall et Mbengue, 2020 ; Mbengue 2019 ; Fall, 2017 ; Diallo, 2013).

La prédominance de la tranche d'âge de 20 à 39 ans (les jeunes) peut être liée au fait que la filière permet de lutter contre le chômage et la pauvreté. Cependant, la colombiculture est une activité qui est dominée dans cette ville de Bambey par la tranche d'âge 15-35ans qui représente $61 \%$ des enquêtés. Les adultes (35- 60 ans) occupent $29 \%$ des colombiculteurs. Les éleveurs dont l'âge dépasse 60 ans sont $4 \%$.

L’âge moyen des aviculteurs avoisine celui observé à Ndjaména au Tchad (Issa et al, 2012) mais il est de 40,44 $\pm 14,23$ ans et est compris entre 19 et 77 ans dans la commune de Thiès (Mbengue, 2019).

La majorité des aviculteurs sont mariés et peut expliquer par la présence des élevages dans les concessions. Ce qui est en phase avec l'élevage de la volaille familiale qui se définit comme la production de volaille à petite échelle pratiquée par des ménages utilisant une main d'œuvre familiale et, autant que faire se peut, les disponibilités alimentaires localement disponibles. Le travail n’est pas rémunéré, et est effectué par les membres de la famille (Sonaiya, 1990).

Le niveau d'instruction des éleveurs qui est de 72,4\% de l'échantillon est en deçà de la moyenne nationale qui est de $80,95 \%{ }^{2}$ en 2018 . Le taux d'analphabète touche plus les femmes car Bambey est une commune rurale où les femmes sont plus représentées dans des activités ménagères. Ces résultats sont contraires à ceux obtenus à Thiès par Fall et al. (2016) qui montrent que tous les aviculteurs de cette ville sont tous instruits. Cependant, la population dans la périphérie de la commune de Thiès qui n’a jamais été dans une école

\footnotetext{
${ }^{2}$ http://uis.unesco.org/fr/country/sn consulté le 18 mars 2020 à 18 h48
} 
est de 5,6\% (Mbengue, 2019) et que le genre est corrélé au niveau d'instruction $\left(\mathrm{X}^{2}=0,09\right)$.

Les professions des éleveurs de volaille vont du fonctionnaire à l'ouvrier en passant par le commerçant, le cultivateur, la ménagère...etc. Les éleveurs de poulets de chair sont dans la plupart des cas des personnes en fonction ou des fonctionnaires retraités qui ont pu épargner pour trouver un capital leur permettant de démarrer leurs activités. L'aviculture se présente comme étant une activité secondaire voire tertiaire pour la plupart des enquêtés car ils ont une profession avec des revenus stables (Gueye, 2002 ; Diallo, 2013 ; Diop, 2016). Au sein de la commune de Thiès, seuls 30,53\% des aviculteurs en font une activité principale par contre 69,74\% la pratiquent comme activité secondaire (Fall et al, 2016). A Dakar qui est la principale zone d'élevage de la volaille, ce sont des pluriactifs qui vont du fonctionnaire à l'ouvrier et qui exercent l'activité. Les des aviculteurs (62\%) avaient une activité professionnelle dans la région de Dakar (Arbelot et al, 1997 ; Arbelot, 1994). De plus, elle présente de bonnes perspectives d'avenir. Ces résultats sont corroborés par les travaux de Mbengue (2019) à Thiès selon lesquels l'aviculture permet de lutter contre le chômage des jeunes en octroyant à ces derniers des revenus satisfaisants.

L'élevage de la volaille est essentiellement une pratique liée à des raisons économiques (Fall et Mbengue, 2021 ; Ouedraogo et al. 2015) à cause des revenus qui peuvent être obtenus mais aussi pour la consommation (Ossebi , 2011). Il est aussi un élevage à cycle court. Cependant, une petite frange des éleveurs de moutons Ladoum de la commune de Thiès au Sénégal (Fall et al, 2017), ont des oies pour assurer une sécurité au sein de leurs exploitations car le vol de bétail reste un réel problème dans cette zone. Il a aussi un rôle d'ordre socio-culturel indéniable car c'est comme un legs, une tradition multiséculaire d'avoir de la volaille au sein de la concession.

Il est à remarquer au sein de cette commune de Bambey que $81 \%$ des colombiculteurs font l'élevage des pigeons par amour (Diop, 2016). Cependant, certains éleveurs sont guidés par des raisons commerciales (15\%) et pour la consommation (4\%).

L'élevage de la volaille est pratiqué depuis plusieurs années car la possession d'animaux est liée aux us et coutumes des populations. L'ancienneté dans l'élevage de volaille est confirmée chez les colombiculteurs de Bambey (Diop , 2016),elle varie entre 1 à 50 ans avec une moyenne de13, $33 \pm 13,6$ ans.

Le manque de formation technique des éleveurs peut être lié à l'absence de projet de développement avicole pour accompagner les aviculteurs et à leur inorganisation professionnelle. 


\subsubsection{Caractéristiques techniques}

Les aviculteurs ont plusieurs espèces et races locales et exotiques de volaille (poulet de chairs, cailles, pigeons, oies, dindes, canards...). Cette stratégie peut être qualifiée de « un peu de tout » (Fall et al, 2016). Elle permet aussi de faire des croisements entre races (Ndeledjel, 2000). Dans les deux types d'élevage (mono et plurispécifiques) le poulet est l'espèce la plus élevée. Ces résultats sont en phase avec ceux observés par Traoré(2006) qui montre que le poulet est l'espèce la plus élevée au sein de l'aviculture dite familiale. Il en est de même au niveau de la province du Sourou, région du nord-ouest Burkinabè, les espèces aviaires élevées sont le poulet, la pintade, le canard en général et accessoirement les pigeons (Ouédraogo et al, 2015).

La présence de plusieurs races d'une même espèce surtout pour les poulets et les pigeons est due le plus souvent à l'amour et l'engouement qu'ont les éleveurs envers ces animaux mais aussi des revenus importants que leur apportent ces derniers.

L'inexistence de la spéculation pondeuse dans les concessions se justifie par le fait qu'elle demande un grand espace, de moyens et une technicité surtout durant les périodes de forte chaleur (Diallo, 2013).

Tous les aviculteurs ont des abris qui sont de dimensions et de fabrications différentes pour leurs volailles car beaucoup sont dans une logique commerciale (Ayssiwede et al, 2013 ; Mbengue , 2019). Cependant à Thiès, les poulaillers sont construits en dur $(100 \%)$ soit avec des toitures en zinc (85\%) ou en fibrociment (15\%) (Fall 2017).

Les éleveurs de poulet de chair utilisent tous des aliments de type industriel à cause de la nature de l'activité dont l'objectif premier est la rentabilité financière. Les autres volailles sont alimentées à base de céréales locales, de restes de cuisine, de pains secs ou avec des aliments industriels.

En dehors des éleveurs qui possèdent des races exotiques (poulets et pigeons), les autres ne donnent pas une grande importance à la reproduction de leur volaille. Cela peut se justifier par le fait que l'espèce hybride ne présente aucune valeur financière surtout quand il s'agit des pigeons. En effet, les colombiculteurs utilisent les pigeons hybrides qu'ils appellent « bâtard » pour la couvaison des œufs des espèces de race pure ou pour « l'embouche » (Diop, 2016).

Les maladies sont assez fréquentes et constituent un handicap pour le développement de cette filière (Ouédraogo et al., 2015). Concernant les poulets de chair, les pathologies les plus récurrentes sont la coccidiose et la salmonellose. Cela est peut-être dû au non-respect des densités dans les élevages mais aussi à l'accès facile dans les poulaillers associés ainsi qu'au manque de moyens de protection sanitaire comme les pédiluves, les masques, bottes ... etc (Diagne , 2008). La maladie de Gumboro est aussi fréquente dans certains élevages. En effet, après la première vaccination au niveau des lieux 
de vente, certains éleveurs préfèrent ne pas effectuer les rappels car jugeant les produits vétérinaires très onéreux. Ils utilisent à la place des complexes vitaminés ou des antibiotiques selon leur propre gré. Le manque de formation de la main d'œuvre, la persistance de la maladie dans la ferme ou la mauvaise conservation des vaccins pourront être des éléments explicatifs (Fall et al, 2016 ; Diallo, 2013).

\subsubsection{Contraintes}

La contrainte majeure des aviculteurs reste la persistance de certaines maladies. Ces dernières sont jugées comme un frein réel au développement de l'aviculture (Mbengue, 2019 ;; Savane, 1996). Ainsi, les pathologies les plus présentes sont la maladie de Newcastle devant celle de Gumboro, la coccidiose, la salmonellose et le coryza. Ces résultats sont conformes avec ceux constatés par Diallo (2013) et Mbengue (2019) au niveau de la commune de Thiès.

L'irrégularité de la qualité de l'aliment et son prix élevé restent une préoccupation majeure (Ndiaye et al., 2007) car la majorité des éleveurs ont de petits effectifs.

La chaleur reste un véritable handicap pour le développement de l'aviculture car les températures diurnes sont assez élevées durant plus de 6 mois.

\section{Conclusion et recommandations}

Il ressort de l'étude que l'aviculture est une activité assez répandue dans la commune de Bambey. Elle mobilise plus d'hommes que de femmes qui sont des mariés et des pluriactifs. L'élevage de la volaille est mené par plusieurs catégories socio-professionnelles comme les fonctionnaires, les retraités, les étudiants, les commerçants, les ouvriers, les ménagères, les agriculteurs et les éleveurs, qui en font une activité secondaire. L'âge des aviculteurs est très variable. Il est à noter une forte présence des jeunes. La motivation des aviculteurs est plus économique car l'activité permet d'avoir des revenus complémentaires.

La typologie fait ressortir deux types d'élevage. Les élevages sont mono spécifiques (poulets ou pigeons) et plurispécifiques (poulets, pigeons, pintades, oies, dindes, canards, cailles). Les races élevées sont très diverses. Cette diversité se rencontre plus au niveau des pigeons et les poulets exotiques. La taille des élevages est très variable et dépend de la spéculation.

Le suivi technique des élevages reste du domaine de l'aviculteur ou de quelques membres de la famille. L'aliment industriel est utilisé pour toutes les espèces élevées mais il est souvent associé avec des céréales locales pour l'alimentation en élevage traditionnel distribué la plupart du temps deux fois par jour. Les éleveurs consacrent beaucoup plus d'importance à la reproduction des pigeons et des poulets de race exotique avec parfois quelques rares croisements. De l'autre côté, avec la divagation des animaux, les éleveurs 
peinent à faire un bon suivi des performances de reproduction et de la couvaison des œufs. Sur le plan sanitaire, la vaccination et le déparasitage ne sont pas systématiques dans certains élevages, ce qui est la cause de la présence des affections comme la maladie de Newcastle, la variole, le coryza, le parasitisme etc. Par ailleurs, des éleveurs font recours à des pratiques empiriques pour la protection ou le traitement de leurs sujets. La commercialisation de la volaille reste problématique au niveau des aviculteurs. Ceendant seuls quelques-uns sollicitent les revendeurs qui sont appelés «Banabanas ».

Cependant, l'aviculture familiale est confrontée à certaines contraintes liées à la cherté de l'aliment industriel, au manque de formation des éleveurs, à la présence et la persistance de certaines maladies, la divagation des animaux cause de prédation et l'absence de foire dans la zone pour l'exposition des différentes espèces élevées. L'inexistence d'agents spécialisés en aviculture et l'inaccessibilité des vaccins constituent des freins pour le développement de cette filière.

Les différents résultats obtenus ont permis de faire quelques recommandations qui sont : (i) étudier d'avantage les performances techniques et économiques de l'élevage urbain de la volaille, (ii) étudier la prévalence de certaines maladies comme la maladie de Gumboro, la maladie de Newcastle, la coccidiose, (iii) faire une étude transversale sur l'aviculture à travers un suivi d'au moins une année.

\section{References:}

1. Agence Nationale Démographie et de la Statistique (ANSD). 2019. Situation économique et sociale du Sénégal en 2016. Ministère de l'Economie et des Finances du Sénégal, Dakar 287p.

2. Arbelot B., Foucher H., Ayon J.F., Missohou A. 1997. Typologie des aviculteurs dans la zone du Cap-Vert au Sénégal. Revue Elev. Med. Vet. Pays Tropicaux, 50(1), 75-83

3. Arbelot B., 1994. Rapport de fin de contrat laboratoire de pathologie aviaire ISRA LNERV - PRODEC 56 janvier 1994 à août 1997.

4. Ayssiwede S.B., Dieng A., Houinato M.R.B., Chrysostome C.A.A.M., Issay., Hornick J.-L., Missohou A. 2013. Elevage des poulets traditionnels ou indigènes au Sénégal et en Afrique Subsaharienne : état des lieux et contraintes. Ann. Méd. Vét. 157, 103-119.

5. Diallo A., 2013. L'élevage de la volaille dans la ville de Thiès, Sénégal : situation et perspectives. Mémoire de fin d'études à l'ISFAR de Bambey. 3p.

6. Diallo A.K., 2018. Caractérisation des exploitations avicoles dans la zone d'intervention du projet ASAN cas des collectivités locales de 
Saré Bidji et de Dioulacolon. Mémoire d'ingénieur des travaux d'élevage ISFAR ex ENCR de Bambey. 53p + annexe.

7. Diop A., 2016. Elevage des pigeons dans la commune de Bambey (Sénégal) : situations et perspectives. Mémoire de fin d'études ISFAR UADB Sénégal 63p.

8. Diagne. M.M., 2008. Analyse de la compétitivité de la filière avicole semi-industrielle dans la zone des Niayes. Mémoire de fin d'études ENSA, Thiès (Sénégal). 60p.

9. Fall. A. K, Mbengue.A.M. 2020. Technical analysis of poultry farming in the municipality of Thies Senegal Agricultural Science Research Journal Volume (10) Issue (12): 342 - 346 December - 2020.

10. Fall. A. K. 2017. L'élevage urbain dans la commune de Thiès au Sénégal : systèmes, caractéristiques socioéconomiques et techniques, perspectives. Thèse de doctorat unique. Université de Thiès, Sénégal

11. Fall A. K, Dieng. A, Ndiaye. S. 2017. L'élevage des moutons de race Ladoum dans la commune de Thiès, Sénégal : caractéristiques socioéconomiques et techniques : Afrique SCIENCE 13(4), 140-150.

12. Fall A., Dieng A., Samba A.N.S., Diallo A., 2016. L'aviculture urbaine familiale au Sénégal : caractérisation et rôle socio-économique dans la commune de Thiès. Rev. CAMES. VOL04. NUM02. ISSN2424-7235 : $07 \mathrm{p}$.

13. Guèye E.F., 2002. Family poultry research and development in lowincome food-deficit countries: approaches and prospects. Outlook on Agriculture, 31(1), 13-21.

14. Issa Y., Mopate L.Y. et Missohou A., 2012. Commercialisation et consommation de la volaille traditionnelle en Afrique subsaharienne. Journal of Animal \& Plant Sciences. Vol. 14, Issue 3: 1985-1995. http://www.m.elewa.org/JAPS; ISSN 2071 - 7024.

15. Mbengue A.M., 2019. Aviculture dans la commune de Thiès (Sénégal) : caractéristiques et contribution dans les revenus des ménages. Mémoire de fin d'étude à l'ISFAR de Bambey. 34, 46, 47p.

16. MEPA, 2019 : Actualisation des paramètres techniques de la filière avicole au Sénégal. Rapport paramètre technique-Aviculture-Final juin 2019.p10, 13.

17. Ndiaye S., Ouedeba B. et Sanders J., 2007. La production avicole intensive au Sénégal : Problématique de l'alimentation et place du sorgho. Bulletin No. 6. October 1, 2007.

18. Ndeledje G.N., 2000. Amélioration génétique de la poule locale au Sénégal par croisement avec les races exotiques : Résultats préliminaires : thèse de doctorat $\mathrm{N}^{\circ} 1$ EIMSV UCAD Dakar $68 \mathrm{p}$.

19. Ouedraogo B., Bale B., Zoundi S. J. et Sawadogo L. 2015 : Caractéristiques de l'aviculture villageoise et influence des techniques 
d'amélioration sur ses performances zootechniques dans la province du Sourou, région Nord-Ouest Burkinabé ; Int. J. Biol. Chem. Sci. 9(3) : 1528-1543. 1538p,

20. Ossebi W. 2011. Analyse de la filière «poulet du pays » au Sénégal : aspects économiques et organisationnels. Mémoire de master II en productions animales et développement durable. $\mathrm{N}^{\circ} 13$ EISMV Dakar Sénégal. $44 \mathrm{p}$

21. PNDE. 2017. Plan National de Développement de L’Elevage. Document $\mathrm{N}^{\circ} 1$. Diagnostic du Secteur de L’Elevage. Ministère de l'Elevage du Sénégal. 30p.

22. Savane M., 1996 : l'aviculture rurale au Sénégal : contraintes et perspectives zoo-économiques cas de la Haute Casamance, thèse de doctorat en Médecine vétérinaire EISMV UCAD Dakar.102p.

23. Sonaiya, E.B, 1990. Poultry husbandry in small rural farms. Entwichlung und landlicher Rawn, 4(90), 3-6.

24. Sonaiya E.B. et Swan S.E.J., 2004. Production en aviculture familiale, un manuel technique. Organisation des Nations Unies pour l'Alimentation et l'Agriculture, Rome, 140.

25. Traoré E.H., 2006. Première évaluation de la structure et de l'importance du secteur avicole commercial et familial en Afrique de l'Ouest : rapport du Sénégal. Organisation des Nations-Unies pour l'Alimentation et l'Agriculture : Rome.52 p.

26. Traoré E.H., 2001. "Système de production aviaire » Projet FAO :»Amélioration de la production avicole en élevage familial traditionnel à travers une meilleure gestion des contraintes de production et de Santé animale TCP/SEN 065». Phase I :2001-2002. Rapport de mission. $36 \mathrm{p}$

29. http ://www.banquemondiale.org/fr/senegal/overview: Sénégal-Vue d'ensemble Banque mondiale (29/03/2020) à 00h 30 